\title{
Frontal and Cerebellar Atrophy Supports FTSD-ALS Clinical Continuum
}

OPEN ACCESS

Edited by:

Andrea Calvo,

University of Turin, Italy

Reviewed by:

Christian Lunetta,

Centro Clinico NEMO, Italy

Francesca Trojsi,

University of Campania Luigi Vanvitelli,

Italy

${ }^{*}$ Correspondence:

Fulvia Palesi

fulvia.palesi@unipvit

†These authors have contributed equally to this work

Received: 10 August 2020 Accepted: 02 November 2020 Published: 26 November 2020

Citation:

Pizzarotti B, Palesi F, Vitali P, Castellazzi G, Anzalone N, Alvisi E, Martinelli D, Bernini S,

Cotta Ramusino M, Ceroni M, Micieli G, Sinforiani E, D'Angelo E, Costa $A$ and

Gandini Wheeler-Kingshott CAM (2020) Frontal and Cerebellar Atrophy Supports FTSD-ALS Clinical Continuum

Front. Aging Neurosci. 12:593526. doi: 10.3389/fnagi.2020.593526
Beatrice Pizzarotti 1,2†, Fulvia Palesi ${ }^{1,3 * \dagger}$, Paolo Vitali ${ }^{4,5}$, Gloria Castellazzi ${ }^{6,7,8}$, Nicoletta Anzalone ${ }^{9}$, Elena Alvisi10, Daniele Martinelli1,11, Sara Bernini ${ }^{12}$, Matteo Cotta Ramusino ${ }^{1,13}$, Mauro Ceroni1,14, Giuseppe Micieli15, Elena Sinforiani12, Egidio D'Angelo ${ }^{1,3}$, Alfredo Costa ${ }^{1,13+}$ and Claudia A. M. Gandini Wheeler-Kingshott1,3,6t

\begin{abstract}
'Department of Brain and Behavioral Sciences, University of Pavia, Pavia, Italy, ${ }^{2}$ Department of Clinical Neuroscience, Lausanne University Hospital and University of Lausanne, Lausanne, Switzerland, ${ }^{3}$ Brain Connectivity Center, IRCCS Mondino Foundation, Pavia, Italy, ${ }^{4}$ Radiology Unit, IRCCS Mondino Foundation, Pavia, Italy, ${ }^{5}$ Department of Radiology, IRCCS Policlinico San Donato, Milan, Italy, ${ }^{6}$ NMR Research Unit, Department of Neuroinflammation, Queen Square MS Centre, UCL Queen Square Institute of Neurology, Faculty of Brain Sciences, University College London, London, United Kingdom, ${ }^{7}$ Department of Electrical, Computer and Biomedical Engineering, University of Pavia, Pavia, Italy, ${ }^{8}$ IRCCS Mondino Foundation, Pavia, Italy, ${ }^{9}$ Neuroradiology Unit, San Raffaele Scientific Institute, Vita-Salute San Raffaele University, Milan, Italy, ${ }^{10}$ Department of Neurology and Laboratory Neuroscience, IRCCS Italian Auxological Institute, Milan, Italy, ${ }^{11}$ Headache Science and Neurorehabilitation, IRCCS Mondino Foundation, Pavia, Italy, ${ }^{12}$ Laboratory of Neuropsychology, IRCCS Mondino Foundation, Pavia, Italy, ${ }^{13}$ Unit of Behavioral Neurology, IRCCS Mondino Foundation, Pavia, Italy, ${ }^{14}$ Department of Neurology, IRCCS Mondino Foundation, Pavia, Italy, ${ }^{15}$ Department of Emergency Neurology, IRCCS Mondino Foundation, Pavia, Italy
\end{abstract}

Background: Frontotemporal Spectrum Disorder (FTSD) and Amyotrophic Lateral Sclerosis (ALS) are neurodegenerative diseases often considered as a continuum from clinical, epidemiologic, and genetic perspectives. We used localized brain volume alterations to evaluate common and specific features of FTSD, FTSD-ALS, and ALS patients to further understand this clinical continuum.

Methods: We used voxel-based morphometry on structural magnetic resonance images to localize volume alterations in group comparisons: patients (20 FTSD, seven FTSD-ALS, and 18 ALS) versus healthy controls (39 CTR), and patient groups between themselves. We used mean whole-brain cortical thickness $(\overline{C T})$ to assess whether its correlations with local brain volume could propose mechanistic explanations of the heterogeneous clinical presentations. We also assessed whether volume reduction can explain cognitive impairment, measured with frontal assessment battery, verbal fluency, and semantic fluency.

Results: Common (mainly frontal) and specific areas with reduced volume were detected between FTSD, FTSD-ALS, and ALS patients, confirming suggestions of a clinical continuum, while at the same time defining morphological specificities for each clinical group (e.g., a difference of cerebral and cerebellar involvement between FTSD and ALS). $\overline{C T}$ values suggested extensive network disruption in the pathological process, with indications of a correlation between cerebral and cerebellar volumes and $\overline{C T}$ in ALS. The analysis of the neuropsychological scores indeed pointed toward 
an important role for the cerebellum, along with fronto-temporal areas, in explaining impairment of executive, and linguistic functions.

Conclusion: We identified common elements that explain the FTSD-ALS clinical continuum, while also identifying specificities of each group, partially explained by different cerebral and cerebellar involvement.

Keywords: ALS, FTD, FTD-ALS continuum, dementia, cerebellum, VBM

\section{INTRODUCTION}

Frontotemporal Spectrum Disorder (FTSD) represents 5\% of all causes of dementia in subjects over 65 years and has two main clinical presentations: the behavioral (bvFTSD) and the linguistic variant (Primary Progressive Aphasia, PPA; Harciarek, 2013). Amyotrophic Lateral Sclerosis (ALS) is a neurodegenerative disease affecting electively the upper and lower motoneuron but several studies have proven that ALS show also cognitive impairment in different domains, like social cognition, verbal memory and executive functions (Leslie et al., 2015). Family forms combining both diseases have already been described, hence FTSD and ALS may be thought as pathophysiological continuum, with up to 50\% ALS patients presenting FTSD symptoms (Lattante et al., 2015). Furthermore, a recent metaanalysis has demonstrated either converging and disease-specific patterns of gray matter atrophy in bvFTSD and ALS patients, supporting the existence of a clinical continuum (Luo et al., 2020). However, it is hard to predict which patients are prone to develop both aspects of the continuum.

Magnetic resonance imaging (MRI) is commonly used to exclude secondary causes of dementia and to detect morphological findings useful for a correct diagnosis. Several voxel based-morphometry (VBM) investigations have used structural MRI images to demonstrate volume alterations in specific areas, such as frontal and temporal lobes, insula, and anterior cingulum, in FTSD-ALS continuum (Christidi et al., 2017; Crespi et al., 2017; Meeter et al., 2017; Cash et al., 2018). Nevertheless, only a few studies looked at cortical thickness as a marker of the FTSD-ALS continuum and mainly focused on ALS patients (Du et al., 2007; Schuster et al., 2014; Hendrikse et al., 2015).

This study aimed to identify the cognitive and neurostructural deterioration of the FTSD-ALS continuum assessing (i) common and specific areas of volume reductions, as identified with VBM, in ALS, FTSD-ALS, and FTSD; (ii) whether whole-brain mean cortical thickness $(\overline{C T}$; as surrogate marker of an overall pathological/functional degeneration) could explain differences between groups and propose possible mechanistic interpretations of the different clinical presentations; and (iii) whether cognitive and neurostructural deterioration are directly related.

Abbreviations: FTSD, Frontotemporal Spectrum Disorder; ALS, Amyotrophic Lateral Sclerosis; CTR, healthy controls; VBM, voxel based-morphometry; CT, cortical thickness; FAB, frontal assessment battery; FAS, verbal fluency; SF, semantic fluency; GM, gray matter; WM, white matter; TIV, total intracranial volume.

\section{MATERIALS AND METHODS}

\section{Subjects}

Forty-five patients belonging to FTSD-ALS continuum were recruited at the IRCCS Mondino Foundation from September 2013 to March 2016. Patients underwent a complete diagnostic workup including neuropsychological assessment, MRI and electroneuromyography, in order to obtain an exhaustive phenotypic profiling and a correct etiological definition. Based on the most recent diagnostic criteria, patients were classified into three etiological groups: FTSD [including bvFTSD (Rascovsky and Grossman, 2013) and PPA (Gorno-Tempini et al., 2011)], ALS (Carvalho and Swash, 2009), and FTSD-ALS. According to Rascovsky diagnostic criteria, FTSD diagnosis was supported, but not determined by the cognitive profile. No patient was excluded on the basis of the neuropsychological profile, if diagnostic criteria were still met. As well, ALS diagnosis was made in patients fulfilling Awaji criteria (Costa et al., 2012), not excluding subjects with a cognitive impairment, as previously. Patients who met both diagnostic criteria were classified in the FTSD-ALS group (Costa et al., 2012; Rascovsky and Grossman, 2013). During the enrollment, MRI was used with prominent exclusion function of secondary causes of cognitive decline.

A group of 39 age- and sex-matched healthy controls (CTR) were selected as a reference group and enrolled on a voluntary basis among subjects attending a local third-age university (University of Pavia, Information Technology course) or included in a program on healthy aging (Fondazione Golgi, Abbiategrasso). All CTR underwent a clinical assessment to exclude any cognitive or motoneuron impairment.

Exclusion criteria included at least one of the following: major psychiatric disorders, pharmacologically treated delirium or hallucinations, secondary causes of cognitive decline (e.g., vascular, metabolic, endocrine, toxic, and iatrogenic).

\section{Standard Protocol Approvals, Registrations, and Patient Consents}

This study was carried out in accordance with the Declaration of Helsinki with written informed consent from all subjects. The protocol was approved by the local ethic committee of the IRCCS Mondino Foundation.

\section{Neuropsychological Assessment}

Forty-three of forty-five patients underwent a complete neuropsychological evaluation including the following cognitive domains: attention (attentive matrices, trail making test $\mathrm{A}$ 
and B, Stroop test), memory (digit span, verbal span, Corsi block-tapping test, logical memory, Rey-Osterrieth complex figure recall, and Rey 15 item test), language [verbal (FAS) and semantic fluency (SF)], executive function [Raven's matrices, Wisconsin card sorting test, and frontal assessment battery (FAB)], and visuo-spatial skills (Rey-Osterrieth complex figure). Cognitive scores were corrected by age and education and compared to the normative cut-off for the Italian population. Two FTSD patients did not undergo the cognitive assessment due to poor collaboration.

Frontal assessment battery, FAS, and SF were selected for the correlation analyses as representative of the executive and linguistic functions usually affected in the FTSD-ALS spectrum.

\section{MRI Acquisition}

All subjects underwent MRI examination within 1 month from cognitive assessment. A standardized MRI protocol was carried out on a Siemens Skyra 3T scanner (Siemens, Erlangen, Germany) with a 32 channel head-coil. A 3D T1-weighted (3DT1w) structural MPRAGE sequence was setup according to the Alzheimer's Disease Neuroimaging Initiative protocol (ADNI2; Jack et al., 2015) with the following parameters: $\mathrm{TR}=2,300 \mathrm{~ms}, \mathrm{TE}=2.95 \mathrm{~ms}, \mathrm{TI}=900 \mathrm{~ms}$, flip angle $=9^{\circ}$, 176 sagittal slices, acquisition matrix $=256 \times 256$, in-plane resolution $=1.05 \times 1.05 \mathrm{~mm}^{2}$, slice thickness $=1.2 \mathrm{~mm}$, and acquisition time $=5.12 \mathrm{~min}$. Standard clinical sequences were performed to exclude other pathologies.

\section{VBM Analysis}

3D T1-weighted images were converted from DICOM to NIFTI format and segmented in their native space into gray matter (GM), white matter (WM), and cerebrospinal fluid (CSF) using the CAT12 (Gaser and Kurth, 2016) Matlab toolbox for SPM12. The segmented images were modulated and normalized to the Montreal Neurological Institute (MNI) space (ICBM-152 template) with $1.5 \mathrm{~mm}$ isotropic voxels, total intracranial volume (TIV) and the mean CT value $(\overline{C T})$ over the whole cortex were assessed with CAT12 (Gaser and Kurth, 2016). The resulting modulated normalized GM and WM images were smoothed using a gaussian kernel of $6 \mathrm{~mm} \times 6 \mathrm{~mm} \times 6 \mathrm{~mm}$ in SPM12 (John, 2013) and were used as inputs for the statistical analysis.

\section{Statistical Analysis}

Demographic and neuropsychologic data were compared using the Statistical Package for the Social Sciences, SPSS21 (IBM, Armonk, NY, United States), to assess significant differences between groups. Gaussian distribution was checked with a Shapiro-Wilk test, then normally distributed variables (age, MMSE and SF) were compared using a one-way ANOVA test with Bonferroni correction, while non-normally distributed ones (FAB and FAS) were compared using a Kruskall-Wallis test (Mann-Whitney for pair comparisons). Categorical variables were compared with a chi-squared test. Two-sided $p<0.05$ was used as significance threshold.

Each group of patients (FTSD, ALS, and FTSD-ALS) was compared voxelwise to the CTR group using a one-way ANOVA VBM analysis, performed with SPM12, to identify the atrophic regions of GM and WM specific for each patient group. The same analysis was carried out between pairs of patient groups.

SPM12 was also used to perform multiple regression analyses on all subjects to correlate GM and WM volume with CT values. For each neuropsychological score, a multiple regression analysis was performed on all patients considered together to determine possible areas responsible for the distribution of results.

For all voxelwise analyses, the significance was set at $p<0.05$ FWE corrected at cluster level. Exploratory results were also investigated with an uncorrected $p<0.001$ together with a cluster extension correction of minimum 160 voxels. Sex, age and TIV were used as covariates.

The XJVIEW toolbox ${ }^{1}$ and FSL anatomical atlases, such as JHU (Oishi et al., 2008) and SUIT (Diedrichsen et al., 2009) were used to accurately localize the regions affected by alterations.

\section{RESULTS}

Overall this study was able to identify specific patterns of volume reduction in ALS, FTSD-ALS, and FTSD patients compared to CTR subjects. Whole brain mean CT was found to correlate with GM and WM volumes, non-necessarily implicated in group differences.

Correlations of volume and neuropsychological scores in the overall patient group indicated that the cerebellum was a key area for the investigated functions, despite atrophy per se was affecting the cerebellum only in the FTSD group.

\section{Patient Characteristics}

Based on clinical criteria, patients were clustered as follows: 20 FTSD (16 bvFTSD and four PPA), 18 ALS and seven patients showing mixed features with FTSD-ALS. Demographic data and cognitive scores are summarized in Table 1.

Etiological groups and CTR were age- and sex-matched. MMSE was significantly reduced in FTSD and FTSD-ALS patients with respect to CTR but did not differ $(p=0.091)$ between the three patient groups (ASL, FTSD, and FTSDALS). FAB scores were homogeneous between patient groups $(p=0.160)$, whereas increasing FAS and SF scores were found from FTSD to ALS group ( $p=0.002$ and $p=0.004$ ).

\section{Comparison Between Patients and Controls}

Voxelwise comparisons between patients and CTR with regard to brain volume reductions are reported in Table 2 . The most compromised group in terms of GM atrophy is the FTSD group, followed by FTSD-ALS and by ALS. In detail, GM regions with reduced volume in FTSD were mainly located (bilaterally) in the frontal and temporal lobes, while WM regions with reduced volume involved several tracts mainly connecting the frontal and the temporal lobes (Figure 1).

Altered regions in FTSD-ALS were lateralized to the left hemisphere and involved GM of the frontal lobe, left insula, and WM of the temporal lobe.

\footnotetext{
${ }^{1}$ http://www.alivelearn.net/xjview/
} 
TABLE 1 | Demographic features and cognitive status.

\begin{tabular}{|c|c|c|c|c|c|}
\hline & $\begin{array}{c}\text { CTR (39) } \\
\text { Mean (SD) }\end{array}$ & $\begin{array}{l}\text { FTSD (20) } \\
\text { Mean (SD) }\end{array}$ & $\begin{array}{c}\text { FTSD-ALS (7) } \\
\text { Mean (SD) }\end{array}$ & $\begin{array}{c}\text { ALS (18) } \\
\text { Mean (SD) }\end{array}$ & $p$-value \\
\hline AGE & 65.85 (10.54) & $66.05(7.74)$ & $69.71(11.21)$ & 63.67 (8.19) & 0.555 \\
\hline MMSE & $27.59(1.50)$ & $21.51(5.27)$ & $20.94(7.88)$ & $25.32(4.11)$ & 0.001 \\
\hline FAB & - & 11.81 (5.59) & $12.93(2.61)$ & $14.12(3.49)$ & $0.160^{\star}$ \\
\hline FAS & - & 13.99 (7.95) & $19.38(12.31)$ & $26.55(9.09)$ & $0.002^{\star}$ \\
\hline
\end{tabular}

Demographic features and cognitive scores for healthy controls (CTR), Frontotemporal Spectrum Disorder (FTSD), Amyotrophic Lateral Sclerosis (ALS), and FTSD-ALS. Values are expresses as mean (SD). P-value refers to significance between all different four groups. Comparisons were performed using one-way ANOVA test with Bonferroni correction, or ${ }^{\dagger}$ Chi-squared test or *Kruskall-Wallis test. MMSE, Mini-Mental State Examination; FAB, Frontal Assessment Battery; FAS, Verbal Fluency; and SF, Semantic Fluency.

TABLE 2 | Regions of reduced volume between different groups of patients and controls.

\begin{tabular}{|c|c|c|c|c|c|c|}
\hline Brain region & & FTSD $<$ CTR & FTSD-ALS $<$ CTR & ALS $<$ CTR $^{\star}$ & FTSD $<$ ALS & FTSD-ALS $<$ ALS \\
\hline \multirow{9}{*}{ Gray matter } & Medial frontal gyrus & BIL & & & & \\
\hline & Precentral gyrus & BIL & & $\mathrm{L}$ & & \\
\hline & Frontal opercular cortex & BIL & L & & & BIL \\
\hline & Frontal pole & $\mathrm{L}$ & & & & \\
\hline & Parahippocampal gyrus & BIL & & & BIL & BIL \\
\hline & Temporal pole & $\mathrm{R}$ & & & $\mathrm{L}$ & BIL \\
\hline & Superior temporal gyrus & & & & & $\mathrm{L}$ \\
\hline & Medial temporal gyrus & & & & $\mathrm{R}$ & L \\
\hline & Inferior temporal gyrus & & & & $\mathrm{R}$ & L \\
\hline & Forceps minor & BIL & & & $\mathrm{R}$ & \\
\hline & Cingulum & BIL & & & & \\
\hline & Anterior thalamic radiation & $\mathrm{L}$ & & & $\mathrm{L}$ & \\
\hline & Superior longitudinal fascic & L & L & & BIL & \\
\hline & Inferior longitudinal fascic & & & & $\mathrm{L}$ & \\
\hline & Uncinate fasciculus & & $L$ & & & \\
\hline \multirow[t]{5}{*}{ Cerebellum } & Crus I & & & & BIL & \\
\hline & Crus II & & & & BIL & \\
\hline & Lobule VIIla & & & & $\mathrm{R}$ & \\
\hline & Lobule VIllb & & & & $\mathrm{R}$ & \\
\hline & Lobule VIIllb & & & & $\mathrm{R}$ & \\
\hline
\end{tabular}

Brain regions with significant volume reduction in patients. Significance was set at $p=0.05$ FWE corrected at cluster-level. ${ }^{*}$ indicates exploratory results at $p=0.001$ with a minimum cluster extent of $k=160$ voxels. The lateralization is identified with: $L$, left; $R$, right; and BIL, bilateral.

Amyotrophic lateral sclerosis did not show any areas with reduced volume; lowering the statistical threshold, though, a reduced GM volume was found in the left pre- and postcentral gyri.

\section{Comparison Between Patient Groups}

Comparisons between atrophic regions (meaning volume reduction of GM or WM) in different groups of patients are also reported in Table 2. Direct comparison between patient groups showed differences when comparing FTSD and FTSD-ALS to ALS patients (Figure 2). FTSD were more atrophic than ALS in several GM temporal areas, in WM regions connecting the frontal and the temporal lobes (the same involved in the comparison with CTR), and extensively in the posterior cerebellum (Crus I/II, lobules VII and VIII).

FTSD-ALS in comparison to FTSD-ALS shared several areas of GM atrophy that emerged in the comparison of 

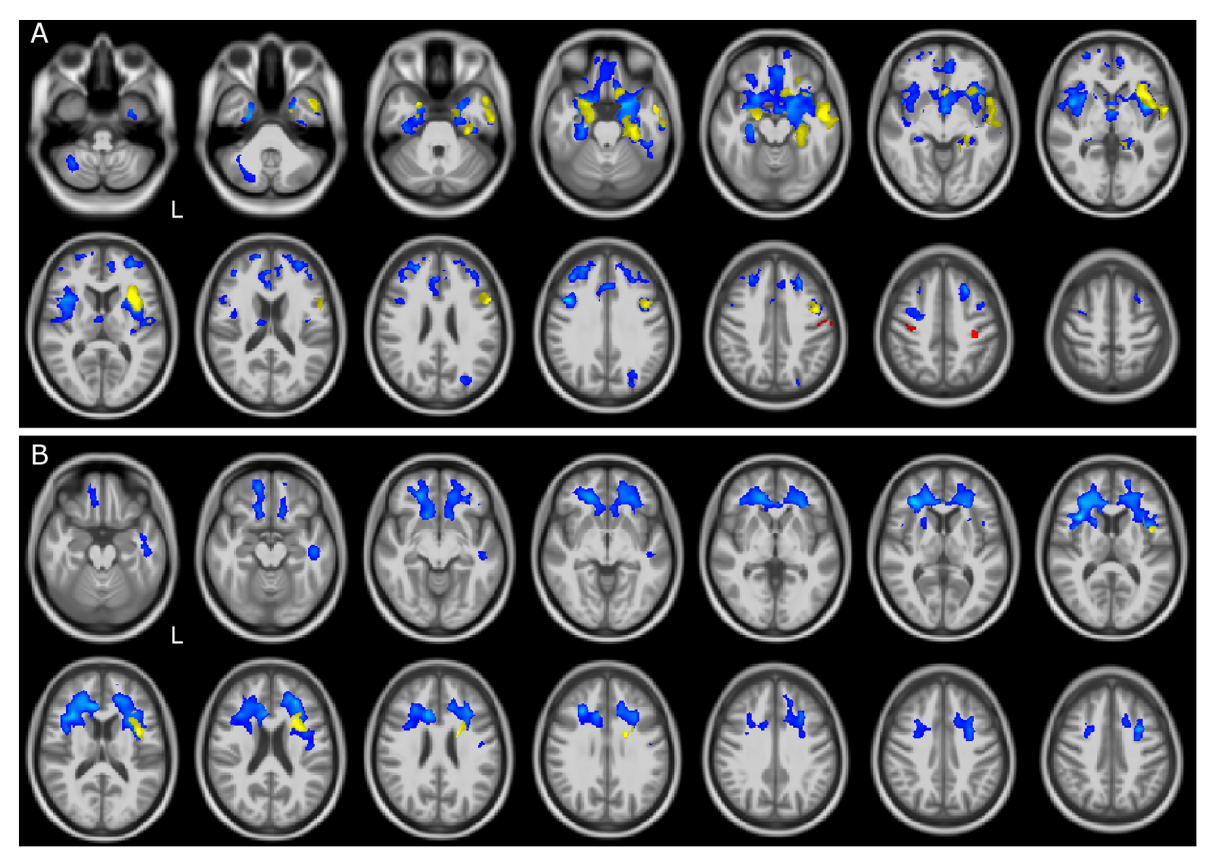

FIGURE 1 | GM and WM volume reduction in patients compared to controls. Regions of gray matter (GM) and white matter (WM) volume reduction in patients compared to controls (CTR). Significance was set at $p<0.05$ FWE corrected at cluster level, except for the comparison ALS and CTR ( $p=0.001, k=160)$. All results are overlaid onto the MNI 152 template and are shown as interleaved axial slices. L indicates the left hemisphere (radiological view). (A) GM atrophic regions in FTSD (blue), FTSD-ALS (yellow), and ALS (red) compared to CTR. (B) WM atrophic regions in FTSD (blue) and FTSD-ALS (yellow) compared to CTR. Blue clusters in (A) are showing that compared to CTR, volume reductions are predominant in FTSD compared to the other forms of disease and are distributed across brain regions involving deep GM and cerebral frontal cortex; yellow clusters are showing that FTSD-ALS patients present lesser involvement of degeneration across brain regions while red clusters are identifying mainly motor areas well known to be affected in ALS. For what concerns WM alterations as shown in (B), blue clusters are predominant and demonstrate a major involvement of pathways in FTSD, while yellow clusters are fewer and more lateralized in FTDS-ALS. No clusters are emerging as altered in ALS.

FTSD to ALS. These areas involved mainly GM of the temporal lobe.

Comparisons of FTSD versus FTSD-ALS did not survive FWE correction. Lowering the statistical threshold, it emerged that in FTSD the insula is the only area with reduced volume than FTSDALS. No WM regions seemed to indicate group specific pattern of volume loss between FTSD and FTSD-ALS and between FTSDALS and ALS.

\section{Correlation Between Volume and CT}

Correlations between volume and whole brain CT in CTR, all patients and different patient groups are reported in the Supplementary Material.

In CTR, positive correlation was found between CT and volume of several GM regions of the frontal, parietal and temporal lobes. In the ALS group, lower CT correlated with lower GM volume in the cerebellum, while lowering the statistical threshold showed that also WM regions connecting frontal and temporal lobes are involved.

\section{Correlation Between Volume and Neuropsychological Scores}

Correlations between neuropsychological scores and volume in all patients are reported in Table 3. Lower
FAB scores correlated with lower GM volume in several cerebellar areas, while no correlations were found in WM regions.

Reduced FAS scores had a significant involvement of GM regions of cerebellum, and of WM volume of multiples subcortical regions.

No correlations were found between SF scores and GM or WM volumes.

\section{DISCUSSION}

The main finding of this study supports the clinical continuum of FTSD, FTSD-ALS, and ALS patients given the presence of shared common structural and cognitive features, but also specific structural features were identified for each etiological group supporting the fact that the clinical continuum is indeed characterized by three different diagnostic entities. The clinical continuum was well detected by fluency scores (both FAS and SF), which were the lowest in FTSD, lower in FTSD-ALS and only slight decreased in ALS with respect to normal scores. The same behavior was detected in volume deterioration: FTSD presented a diffuse cerebral (bilateral frontotemporal) and cerebellar atrophy, FTSD-ALS presented a less pronounced cerebral (left frontotemporal) and 

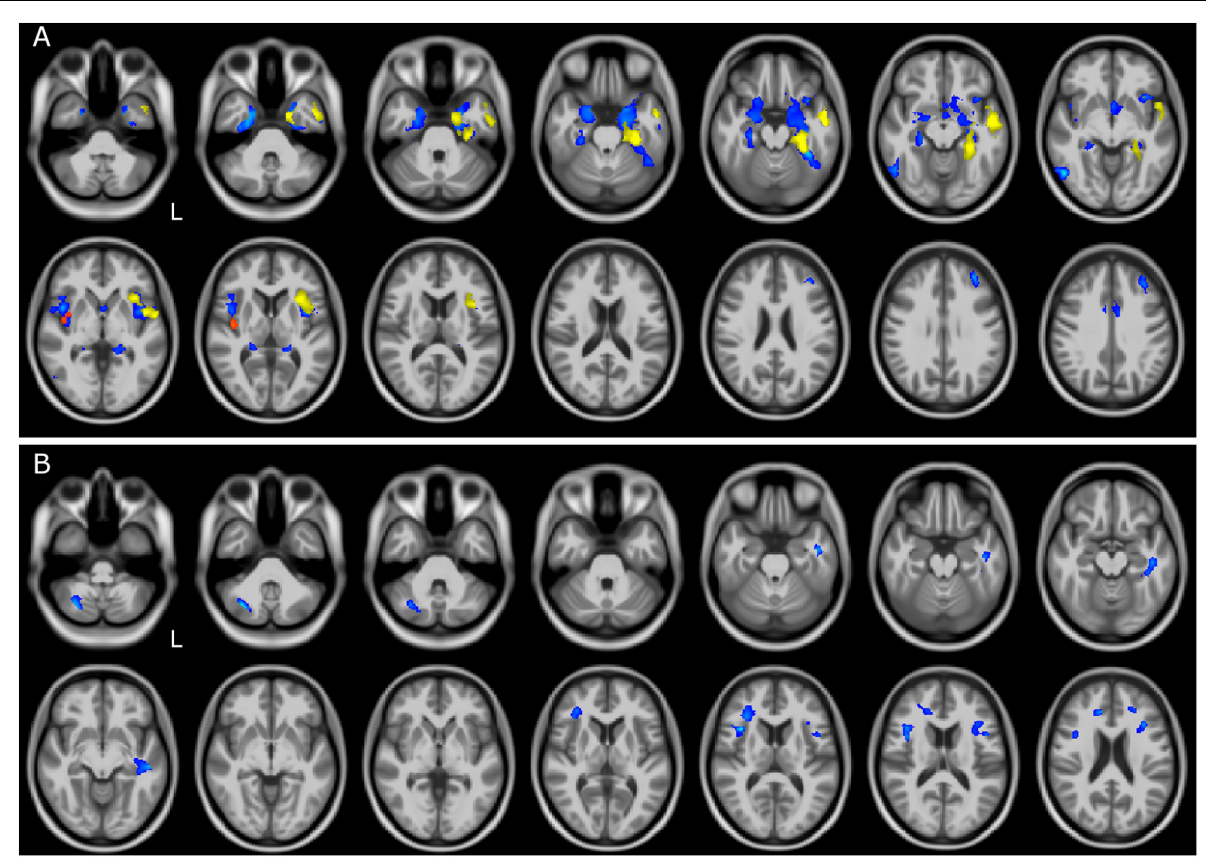

FIGURE 2 | GM and WM volume reduction between patient groups. Regions of gray matter (GM) and white matter (WM) volume reduction between patients. Significance was set at $p<0.05$ FWE corrected at cluster level, except for the comparison FTSD and FTSD-ALS $(p=0.001, k=160)$. All results are overlaid onto the MNI 152 template and are shown as interleaved axial slices. L indicates the left hemisphere (radiological view). (A) GM atrophic regions in FTSD vs ALS (blue), FTSD-ALS vs ALS (yellow), and FTSD vs FTSD-ALS (red). (B) WM atrophic regions in FTSD (blue) compared to ALS. Differences between groups are demonstrating both GM and WM volume reductions in FTSD compared to ALS, as shown by blue clusters in (A, B). There are also a number of yellow clusters in (A) identifying more severe GM volume reductions in FTSD-ALS compared to ALS patients, while no differences were detected in WM between these two groups as shown by the lack of yellow clusters in (B). Differences in terms of volume reduction between FTSD and FTSD-ALS were localized to a lateralized cluster of GM as shown in (A).

cerebellar atrophy, while ALS presented a minimal atrophy (bilateral pericentral).

Interestingly, however, there are clear specificities showing involvement of cognitive areas and of WM disruption that contribute to differentiate clinical and neuropsychological presentations. Common features included more atrophic

TABLE 3 | Neuropsychological regression analysis.

\begin{tabular}{|c|c|c|c|c|}
\hline Brain region & & FAB & FAS & SF \\
\hline \multirow[t]{3}{*}{ White matter } & SLF (frontal/temporal part) & & $\mathrm{R}$ & \\
\hline & SLF (parietal part) & & $L$ & \\
\hline & Anterior thalamic radiation & & $\mathrm{L}$ & \\
\hline \multirow[t]{8}{*}{ Cerebellum } & Lobule IV & & BIL & \\
\hline & Lobule V & & $\mathrm{R}$ & \\
\hline & Lobule VI & & BIL & \\
\hline & Crus I & $L$ & BIL & \\
\hline & Crus ॥ & $L$ & BIL & \\
\hline & Lobule VII & $\mathrm{L}$ & BIL & \\
\hline & Lobule VIII & $L$ & BIL & \\
\hline & Lobule IX & & $\mathrm{L}$ & \\
\hline
\end{tabular}

Areas of positive correlation between neuropsychological scores and brain volume. Significance was set at $p=0.05$ FWE corrected at cluster-level. The lateralization is identified with: L, left; R, right; BIL, bilateral; and SLF, superior longitudinal fasciculus. frontal lobes compared to CTR. It is noteworthy that while areas of GM atrophy were found in all three groups of patients compared to CTR, WM atrophy was more disease specific, with extensive involvement in FTSD and some involvement in FTSD-ALS.

Atrophy of frontal and temporal cortices in FTSD patients confirms previous results (Kanda et al., 2008; Luo et al., 2020), whereas spread reduced WM volumes indicates an overall network disruption that may be independent or secondary to GM atrophy. The present cross-sectional data cannot answer mechanistic questions on WM and GM alterations in FTSD patients, that need to be dealt with appropriate dedicated longitudinal studies where the interplay of GM and WM involvement can be followed over time. The finding that also WM is significantly affected is in line with recent histopathologic studies that hypothesize a cortico-efferent pathway of disease spreading, given the progressive distribution of phosphorylated $43-\mathrm{kDA}$ TAR DNAbinding protein (pTDP-43) in different brain regions in both FTSD patients (Brettschneider et al., 2014) and ALS patients (Braak et al., 2013).

FTSD-ALS patients, instead, showed lateralized alterations (to the left hemisphere) in the same frontal and temporal GM areas that are also involved in FTSD. Given that the insula has a pivotal role in cognitive functions (self-perception, motivation, executive functions, and subjective responses) 
and the anterior insula is connected with dorsolateral and ventromedial prefrontal cortex (Namkung et al., 2017) it is interesting that this brain region is more and bilaterally atrophic in the FTSD group with worse executive functions. In addition, the FTSD-ALS group showed involvement of some WM regions belonging to the superior longitudinal fasciculus (SLF), also altered in FTSD, as well as of the uncinate fasciculus (UF). It is to note that the involvement of SLF and UF was previously detected also in different cohorts of ALS patients using tractography and tract based spatial statistics (TBSS), which revealed alteration of standard diffusion-derived metrics, such as fractional anisotropy, radial and axial diffusivity (Cirillo et al., 2012; Christidi et al., 2017). The SLF and UF are both associative long tracts that connect different lobes of the brain, with the SLF being known to contribute to higher motor functions while the UF has a role in memory and emotional behavior (Von Der Heide et al., 2013), their involvement, therefore, supports the mixed clinical presentation of FTSD-ALS patients and the existence of a clinical continuum.

In ALS patients, previous studies reported atrophy in non-motor areas involved in executive and behavioral functions, such as frontal, temporal and limbic regions (Menke et al., 2014; Luo et al., 2020). Although our ALS patients did not show atrophy in those regions, the involvement of motor and premotor regions emerging from a less stringent statistical analysis is indeed consistent with motor symptoms onset in ALS.

In order to assess potential functional implications of the atrophic areas, we performed a post hoc analysis of our results evaluating whether the changes could be captured in terms of resting state networks (see Supplementary Materials). We classified all those voxels belonging to atrophic regions that spatially overlapped standard resting state networks for more than $10 \%$ of their volume. Thus, the ALS group showed an involvement of the sensory motor network (SMN; Tedeschi et al., 2012; Agosta et al., 2013), whereas FTSD-ALS showed atrophy spread across motor (e.g., frontal cortex), sensory (e.g., occipital visual network), and associative networks, including the default mode network (DMN) although limited to the hippocampus. Intriguing is the lack of volume reduction in motor areas in the FTSD-ALS group, which is captured also by the absence of SMN involvement. This is probably due to the statistical power of the VBM analysis as the precentral gyrus is different between FTSD-ALS and CTR but does not survive multiple comparisons. In FTSD there was a widespread involvement not only of sensory and associative networks, but of all cognitive domains including executive function networks, DMN and cerebellar network. As previously suggested (Trojsi et al., 2014) these three groups of patients can be considered as a clinical continuum, where subjects belong to one group or the other depending on the domain affected by tissue atrophy.

Furthermore, the direct comparison between patients highlighted that some regions of the temporal lobe had reduced volume in FTSD and FTSD-ALS compared to ALS. Interestingly, FTSD showed reduced cerebellar volume compared to ALS, which confirms findings of previous studies in C9orf72 mutated patients (Tan et al., 2014). Genetic data were not available for our analysis, but it would be interesting to understand whether cerebellar involvement is gene-dependent. Moreover, since our FTSD group was mainly represented by the behavioral variant (16 subjects), we could also hypothesize that cerebellar alterations, which were shown in this group, are particularly relevant to this disease phenotype. The fact that cerebellar Crus I/II (bilaterally) was involved in FTSD compared to ALS, could partially explain the cognitive impairment of these patients given the cerebellar role in memory, language processing and executive functions (Habas et al., 2009; Gellersen et al., 2017) as well in continuous cognitive processing tasks (Castellazzi et al., 2018). Interestingly, another study showed the involvement of postero-lateral cerebellum in psychomotor speed and working memory, particularly in differentiating two similar experiences (Shiroma et al., 2016). Furthermore, our study shows an involvement of the posterior cerebellum in FTSD compared to ALS, which could point to a greater disruption of the cerebro-cerebellar circuit in FTSD. This is further supported by the reduced volume of the anterior thalamic radiation, which is known to be part of the efferent pathway from the superior cerebellar peduncle (Palesi et al., 2015).

In order to understand the source of atrophy in the three patient groups and help mechanistic interpretation of the VBM results, we investigated the correlation between $\overline{C T}$ values and volume, as measured by VBM analysis. Indeed, both VBM volume changes and cortical thickness measurements are based on cortex morphology, but with cortical thickness being more specific to cellular density. Details of $\overline{C T}$ correlations with local volumes are given in the Supplementary Materials; nevertheless, it is worth noting that the correlation between $\overline{C T}$ and local volume of long WM bundles in ALS, could suggest a key role of inter-lobe WM integrity for cognitive functions. Alterations of $\overline{C T}$ and temporal WM in FTSD, instead, is consistent with emotional and cognitive impairment in this group of patients.

The correlation between neuropsychological scores and brain volume was performed to elucidate whether the cognitive involvement could be described in terms of atrophy of specific brain regions. The correlation between neuropsychological scores and brain volume for the overall patient group is consistent with recent literature showing more and more often that the cerebellum has a key role in cognition and in supporting advanced functions (Castellazzi et al., 2018). Furthermore, recent studies have reported the presence of a high proportion of cerebellar connections with the frontal and prefrontal cortex (Palesi et al., 2017) consistent with the fact that the FAB is thought to require predominantly frontal and prefrontal cortex and more generally high-level functions. Indeed, Crus I is known to be involved in cognition, whereas lobule VII has recently been shown to have a role in cognitive and social behavior, with particular focus on persisting behavior and novelty seeking (Badura et al., 2018). Since the cerebellar areas correlating with $\mathrm{FAB}$ are also those resulting more atrophic in FTSD compared to ALS (i.e., Crus I/II and lobule 
VII/VIII), it is possible that the correlation between cerebellar volume and neuropsychological scores is driven by alterations of the FTSD group. Future studies will be able to confirm the generalization of these results for the FAB test. Our findings also revealed that lower performances of the verbal fluency test, i.e., FAS, correlated with reduced volume of both frontal areas, consistently with their inhibitory role, and with mostly bilateral cerebellar areas, including Crus I/II as well as both the anterior (lobule IV, V, and VI) and posterior (lobule VII, VIII, and IX) cerebellum. The extensive cerebellar involvement can be explained by the amnestic and linguistic roles of Crus I/II and by the motor involvement of the anterior cerebellum (Gellersen et al., 2017).

These interesting results, however, must be interpreted with caution. The relatively small number of patients per group, in particular for FTSD-ALS, may have reduced the statistical power of some analysis; moreover, within groups there were possible sub-groups like in FTSD where both linguistic and behavioral variants were included together, potentially reducing sensitivity to detect further significant differences. Nonetheless, it is important to consider that FTSD and ALS are rare diseases so larger cohorts may be feasible in future multi-center studies. Unfortunately, any genetic data was available in our cohort, thus we were not able to infer any association between a specific neuropsychological and/or imaging pattern with genetic profile. Another consideration is the absence of ALS-specific neuropsychologic assessment, where motor impairment is taken into account as it might affect the ability to perform a complete cognitive exam (Abrahams et al., 2014). Nonetheless, our decision was driven by the fact that all ALS patients were able to complete the neuropsychologic evaluation.

The CTR group did not undergo the neuropsychological examination, therefore limiting the correlation analysis to patients. Having CTR scores would be highly desirable for future studies.

\section{CONCLUSION}

In conclusion, our study assessed morphological alterations of FTSD, FTSD-ALS, and ALS patients in the attempt to clarify the substrate of known clinical differences and their clinical continuum. The involvement of GM areas, to different extent, in frontal regions in all groups, with atrophy of insular areas in FTSD and FTSD-ALS patients, and temporal cortices and WM regions in FTSD only, supports the presence of shared features, but, at the same time, very distinctive characteristics of these patient groups. Interestingly, cerebellar differences between FTSD and ALS as well as the cerebellar role in correlations between volume and cognitive scores, indicates that the cerebellum contributes to determining the FTSD or ALS variant of this continuum. Future longitudinal multi-center studies are needed to better investigate the relation between localized volume reduction, clinical and neuropsychological outcomes, and $\overline{C T}$ in terms of mechanisms of the FTSD and ALS spectrum.

\section{DATA AVAILABILITY STATEMENT}

The raw data supporting the conclusions of this article will be made available by the authors, without undue reservation.

\section{ETHICS STATEMENT}

The studies involving human participants were reviewed and approved by IRCCS Mondino Foundation. The patients/participants provided their written informed consent to participate in this study.

\section{AUTHOR CONTRIBUTIONS}

BP, FP, CGWK, and AC conceptualized and discussed the study, with contribution from GM and ED'A. BP and FP designed and performed the analyses. FP, PV, GC, and CGWK designed the MRI protocol. PV and NA performed MRI acquisition and neuroradiological evaluation. GC and DM performed the analysis of resting state networks. EA, MCR, MC, and AC performed the clinical assessment and patients recruitment. SB and ES performed the neuropsychological assessment. BP, FP, CGWK, and $\mathrm{AC}$ coordinated the project. BP, FP, MCR, ED'A, CGWK, and $\mathrm{AC}$ wrote the manuscript, with comments from all other authors. All authors contributed to the article and approved the submitted version.

\section{FUNDING}

This work was performed at the IRCCS Mondino Foundation and was supported by the Italian Ministry of Health (RC20142017). FP and ED'A received funding from the European Union's Horizon 2020 Framework Program for Research and Innovation under the Specific Grant Agreement No. 785907 (Human Brain Project SGA2) and No. 945539 (Human Brain Project SGA3). The United States Multiple Sclerosis Society and UCL-UCLH Biomedical Research Centre for ongoing support of the Queen Square MS Centre (CGWK). CGWK receives funding from ISRT, Wings for Life, and the Craig H. Neilsen Foundation (the INSPIRED study), from the MS Society (\#77), Wings for Life (\#169111), and Horizon2020 (CDS-QUAMRI, \#634541).

\section{ACKNOWLEDGMENTS}

We thank the patients, their families, all healthy volunteers for making this research possible. We thank Giancarlo Germani for MRI acquisitions and Roberta Fortunato for her support to the study organization.

\section{SUPPLEMENTARY MATERIAL}

The Supplementary Material for this article can be found online at: $\quad$ https://www.frontiersin.org/articles/10.3389/fnagi.2020. 593526/full\#supplementary-material 


\section{REFERENCES}

Abrahams, S., Newton, J., Niven, E., Foley, J., and Bak, T. H. (2014). Screening for cognition and behaviour changes in ALS. Amyotr. Later. Scler. Frontotemp. Degen. 15, 9-14. doi: 10.3109/21678421.2013.8 05784

Agosta, F., Canu, E., Valsasina, P., Riva, N., Prelle, A., Comi, G., et al. (2013). Divergent brain network connectivity in amyotrophic lateral sclerosis. Neurobiol. Aging 34, 419-427. doi: 10.1016/j.neurobiolaging.2012. 04.015

Badura, A., Verpeut, J. L., and Wang, S. S.-H. (2018). Normal cognitive and social development require posterior cerebellar activity. eLife 7:e36401. doi: 10.7554/ eLife.36401.001

Braak, H., Brettschneider, J., Ludolph, A. C., Lee, V. M., Trojanowski, J. Q., and Tredici, K. D. (2013). Amyotrophic lateral sclerosis-a model of corticofugal axonal spread. Nat. Rev. Neurol. 9, 708-714. doi: 10.1038/nrneurol. 2013.221

Brettschneider, J., Del Tredici, K., Irwin, D. J., Grossman, M., Robinson, J. L., Toledo, J. B., et al. (2014). Sequential distribution of pTDP-43 pathology in behavioral variant frontotemporal dementia (bvFTD). Acta Neuropathol. 127, 423-439. doi: 10.1007/s00401-013-1238-y

Carvalho, M. D., and Swash, M. (2009). Awaji diagnostic algorithm increases sensitivity of El Escorial criteria for ALS diagnosis. Amyotroph. Lateral Scler. 10, 53-57. doi: 10.1080/17482960802521126

Cash, D. M., Bocchetta, M., Thomas, D. L., Dick, K. M., Van Swieten, J. C., Borroni, B., et al. (2018). Patterns of gray matter atrophy in genetic frontotemporal dementia: results from the GENFI study. Neurobiol. Aging 62, 191-196. doi: 10.1016/j.neurobiolaging.2017.10.008

Castellazzi, G., Bruno, S. D., Toosy, A. T., Casiraghi, L., Palesi, F., Savini, G., et al. (2018). Prominent changes in cerebro-cerebellar functional connectivity during continuous cognitive processing. Front. Cell. Neurosci. 12:331. doi: 10.3389/ fncel.2018.00331

Christidi, F., Karavasilis, E., Riederer, F., Zalonis, I., Ferentinos, P., Velonakis, G., et al. (2017). Gray matter and white matter changes in non-demented amyotrophic lateral sclerosis patients with or without cognitive impairment: a combined voxel-based morphometry and tract-based spatial statistics wholebrain analysis. Brain Imaging Behav. 12, 547-563. doi: 10.1007/s11682-0179722-y

Cirillo, M., Esposito, F., Tedeschi, G., Caiazzo, G., Sagnelli, A., Piccirillo, G., et al. (2012). Widespread microstructural white matter involvement in amyotrophic lateral sclerosis: a whole-brain DTI study. AJNR Am. J. Neuroradiol. 33, 1102 1108. doi: 10.3174/ajnr.A2918

Costa, J., Swash, M., and de Carvalho, M. (2012). Awaji criteria for the diagnosis of amyotrophic lateral sclerosis:a systematic review. Arch. Neurol. 69, 1410-1416. doi: 10.1001/archneurol.2012.254

Crespi, C., Dodich, A., Cappa, S. F., Canessa, N., Iannaccone, S., Corbo, M., et al. (2017). Multimodal MRI quantification of the common neurostructural bases within the FTD-ALS continuum. Neurobiol. Aging 62, 95-104. doi: 10.1016/j. neurobiolaging.2017.09.019

Diedrichsen, J., Balsters, J. H., Flavell, J., Cussans, E., and Ramnani, N. (2009). A probabilistic MR atlas of the human cerebellum. NeuroImage 46, 39-46. doi: 10.1016/j.neuroimage.2009.01.045

Du, A. T., Schuff, N., Kramer, J. H., Rosen, H. J., Gorno-Tempini, M. L., and Rankin, K. (2007). Different regional patterns of cortical thinning in Alzheinr's disease and frontotemporal dementia. Brain 130, 1150-1166. doi: 10.1017/ S1368980009991996. Validation

Gaser, C., and Kurth, F. (2016). Manual Computational Anatomy Toolbox-CAT12. Jena: University of Jena.

Gellersen, H. M., Guo, C. C., O'Callaghan, C., Tan, R. H., Sami, S., and Hornberger, M. (2017). Cerebellar atrophy in neurodegeneration-a metaanalysis. J. Neurol. Neurosurg. Psychiatry 88, 780-788. doi: 10.1136/jnnp-2017315607

Gorno-Tempini, M. L., Rascovsky, K., Knopman, D. S., Boeve, B. F., Cappa, S. F., Hodges, J. R., et al. (2011). Classification of primary progressive aphasia and its variants. Neurology 76, 1006-1014.

Habas, C., Kamdar, N., Nguyen, D., Prater, K., Beckmann, C. F., Menon, V., et al. (2009). Distinct cerebellar contributions to intrinsic connectivity networks. J. Neurosci. 29, 8586-8594. doi: 10.1523/JNEUROSCI.1868-09. 2009

Harciarek, S. C. M. (2013). Language, executive function and social cognition in the diagnosis of frontotemporal dementia syndromes. Int. Rev. Psychiatry 25, 178-196. doi: 10.1002/aur.1474. Replication

Hendrikse, J., Van Den Berg, L. H., Walhout, R., Westeneng, H. J., Van De Heuvel, M. P., Veldnick, J. H., et al. (2015). Cortical thickness in ALS: towards a marker for upper motor neuron involvement. J. Neurol. Neurosurg. Psychiatry 86, 288-294. doi: 10.1136/jnnp-2013-30 6839

Jack, C. R., Barnes, J., Bernstein, M. A., Borowski, B. J., Brewer, J., Clegg, S., et al. (2015). Magnetic resonance imaging in Alzheimer's disease neuroimaging initiative 2. Alzheimers Dement. 11, 740-756. doi: 10.1016/j.jalz.2015. 05.002

John, A. (2013). Generative models for MRI/DWI. Front. Neuroinform. 7:13. doi: 10.3389/conf.fninf.2013.10.00013

Kanda, T., Ishii, K., Uemura, T., Miyamoto, N., Yoshikawa, T., Kono, A. K., et al. (2008). Comparison of grey matter and metabolic reductions in frontotemporal dementia using FDG-PET and voxel-based morphometric MR studies. Eur. J. Nucl. Med. Mol. Imaging 35, 2227-2234. doi: 10.1007/s00259-0080871-5

Lattante, S., Ciura, S., Rouleau, G. A., and Kabashi, E. (2015). Defining the genetic connection linking amyotrophic lateral sclerosis (ALS) with frontotemporal dementia (FTD). Trends Genet. 31, 263-273. doi: 10.1016/j.tig.2015. 03.005

Leslie, F. V. C., Hsieh, S., Caga, J., Savage, S. A., Mioshi, E., Hornberger, M., et al. (2015). Semantic deficits in amyotrophic lateral sclerosis. Amyotr. Later. Scler. Frontotemp. Degen. 16, 46-53. doi: 10.3109/21678421.2014.98 7301

Luo, C., Hu, N., Xiao, Y., Zhang, W., Gong, Q., and Lui, S. (2020). Comparison of gray matter atrophy in behavioral variant frontal temporal dementia and amyotrophic lateral sclerosis: a coordinate-based meta-analysis. Front. Aging Neurosci. 12:14. doi: 10.3389/fnagi.2020.00014

Meeter, L. H., Kaat, L. D., Rohrer, J. D., and Van Swieten, J. C. (2017). Imaging and fluid biomarkers in frontotemporal dementia. Nat. Rev. Neurol. 13, 406-419. doi: 10.1038/nrneurol.2017.75

Menke, R. A. L., Körner, S., Filippini, N., Douaud, G., Knight, S., Talbot, K. et al. (2014). Widespread grey matter pathology dominates the longitudinal cerebral MRI and clinical landscape of amyotrophic lateral sclerosis. Brain 137, 2546-2555. doi: 10.1093/brain/awu162

Namkung, H., Kim, S.-H., and Sawa, A. (2017). The insula: an underestimated brain area in clinical neuroscience, psychiatry, and neurology. Trends Neurosci. 40, 200-207. doi: 10.1016/j.tins.2017.02.002

Oishi, K., Zilles, K., Amunts, K., Faria, A., Jiang, H., Li, X., et al. (2008). Human brain white matter atlas: identification and assignment of common anatomical structures in superficial white matter. NeuroImage 43, 447-457. doi: 10.1016/j. neuroimage.2008.07.009

Palesi, F., Rinaldis, A., Castellazzi, G., Calamante, F., Muhlert, N., Chard, D., et al. (2017). Contralateral cortico-ponto- cerebellar pathways reconstruction in humans in vivo: implications for reciprocal cerebro-cerebellar structural connectivity in motor and non-motor areas. Sci. Rep. 7:12841. doi: 10.1038/ s41598-017-13079-8

Palesi, F., Tournier, J. D., Calamante, F., Muhlert, N., Castellazzi, G., Chard, D. et al. (2015). Contralateral cerebello-thalamo-cortical pathways with prominent involvement of associative areas in humans in vivo. Brain Struct. Funct. 220, 3369-3384. doi: 10.1007/s00429-014-0861-2

Rascovsky, K., and Grossman, M. (2013). Clinical diagnostic criteria and classification controversies in frontotemporal lobar degeneration. Int. Rev. Psychiatry 25, 145-158. doi: 10.3109/09540261.2013.763341

Schuster, C., Kasper, E., Dyrba, M., Machts, J., Bittner, D., Kaufmann, J., et al. (2014). Cortical thinning and its relation to cognition in amyotrophic lateral sclerosis. Neurobiol. Aging 35, 240-246. doi: 10.1016/j.neurobiolaging.2013. 07.020

Shiroma, A., Nishimura, M., Nagamine, H., Miyagi, T., Hokama, Y., Watanabe, T., et al. (2016). Cerebellar contribution to pattern separation of human hippocampal memory circuits. Cerebellum 15, 645-662. doi: 10.1007/s12311015-0726-0 
Tan, R. H., Devenney, E., Dobson-Stone, C., Kwok, J. B., Hodges, J. R., Kiernan, M. C., et al. (2014). Cerebellar integrity in the amyotrophic lateral sclerosis-frontotemporal dementia continuum. PLoS One 9:e105632. doi: 10.1371/journal.pone.010 5632

Tedeschi, G., Trojsi, F., Tessitore, A., Corbo, D., Sagnelli, A., Paccone, A., et al. (2012). Interaction between aging and neurodegeneration in amyotrophic lateral sclerosis. Neurobiol. Aging 33, 886-898. doi: 10.1016/j.neurobiolaging. 2010.07.011

Trojsi, F., Esposito, F., de Stefano, M., Buonanno, D., Conforti, F. L., Corbo, D., et al. (2014). Functional overlap and divergence between ALS and bvFTD. Neurobiol. Aging 36, 413-423. doi: 10.1016/j.neurobiolaging.2014. 06.025

Von Der, Heide, R. J., Skipper, L. M., Klobusicky, E., and Olson, I. R. (2013). Dissecting the uncinate fasciculus: disorders, controversies and a hypothesis. Brain 136, 1692-1707. doi: 10.1093/brain/ awt094

Conflict of Interest: The authors declare that the research was conducted in the absence of any commercial or financial relationships that could be construed as a potential conflict of interest.

Copyright (c) 2020 Pizzarotti, Palesi, Vitali, Castellazzi, Anzalone, Alvisi, Martinelli, Bernini, Cotta Ramusino, Ceroni, Micieli, Sinforiani, D'Angelo, Costa and Gandini Wheeler-Kingshott. This is an open-access article distributed under the terms of the Creative Commons Attribution License (CC BY). The use, distribution or reproduction in other forums is permitted, provided the original author(s) and the copyright owner(s) are credited and that the original publication in this journal is cited, in accordance with accepted academic practice. No use, distribution or reproduction is permitted which does not comply with these terms. 